\title{
ON ABSOLUTE MATRIX SUMMABILITY METHODS
}

\author{
H. S. ÖZARSLAN AND H. N. ÖĞDÜK
}

Received 27 October 2004 and in revised form 1 August 2005

We have proved a theorem on $\left|T, p_{n}\right|_{k}$ summability methods. This theorem includes a known theorem.

\section{Introduction}

Let $\sum a_{n}$ be a given infinite series with partial sums $\left(s_{n}\right)$. By $\left(w_{n}^{\delta}\right)$, we denote the $n$th Cesàro means of order $\delta(\delta>-1)$ of the sequence $\left(s_{n}\right)$. The series $\sum a_{n}$ is said to be summable $|C, \delta|_{k}, k \geq 1$, if (see [3])

$$
\sum_{n=1}^{\infty} n^{k-1}\left|w_{n}^{\delta}-w_{n-1}^{\delta}\right|^{k}<\infty
$$

In the special case for $\delta=1,|C, \delta|_{k}$ summability reduces to $|C, 1|_{k}$ summability.

Let $\left(p_{n}\right)$ be a sequence of positive numbers such that

$$
P_{n}=\sum_{v=0}^{n} p_{v} \longrightarrow \infty \quad \text { as } n \longrightarrow \infty, \quad\left(P_{-i}=p_{-i}=0, i \geq 1\right)
$$

The sequence-to-sequence transformation

$$
\vartheta_{n}=\frac{1}{P_{n}} \sum_{v=0}^{n} p_{v} s_{v}
$$

defines the sequence $\left(\vartheta_{n}\right)$ of the $\left(\bar{N}, p_{n}\right)$ means of the sequence $\left(s_{n}\right)$, generated by the sequence of coefficients $\left(p_{n}\right)$ (see [4]). The series $\sum a_{n}$ is said to be summable $\left|\bar{N}, p_{n}\right|_{k}$, $k \geq 1$, if (see [1])

$$
\sum_{n=1}^{\infty}\left(\frac{P_{n}}{p_{n}}\right)^{k-1}\left|\vartheta_{n}-\vartheta_{n-1}\right|^{k}<\infty
$$

If we take $p_{n}=1$ for all values of $n$, then $\left|\bar{N}, p_{n}\right|_{k}$ summability is the same as $|C, 1|_{k}$ summability. 
2518 On absolute matrix summability methods

Given a normal matrix $T=\left(t_{n k}\right)$, we associate two lower semimatrices $\bar{T}=\left(\bar{t}_{n k}\right)$ and $\widehat{T}=\left(\hat{t}_{n k}\right)$ as follows:

$$
\begin{gathered}
\bar{t}_{n k}=\sum_{i=k}^{n} t_{n i}, \quad n, k=0,1, \ldots, \\
\hat{t}_{00}=\bar{t}_{00}=t_{00}, \quad \hat{t}_{n k}=\bar{t}_{n k}-\bar{t}_{n-1, k}, \quad n=1,2, \ldots
\end{gathered}
$$

It may be noted that $\bar{T}$ and $\hat{T}$ are the well-known matrices of series-to-sequence and series-to-series transformations, respectively. Then, we have

$$
\begin{aligned}
T_{n}(s) & =\sum_{v=0}^{n} t_{n v} s_{v}=\sum_{v=0}^{n} \bar{t}_{n v} a_{v} \\
\bar{\Delta} T_{n}(s) & =\sum_{v=0}^{n} \hat{t}_{n v} a_{v} .
\end{aligned}
$$

The series $\sum a_{n}$ is said to be summable $\left|T, p_{n}\right|_{k}, k \geq 1$, if (see [5])

$$
\sum_{n=1}^{\infty}\left(\frac{P_{n}}{p_{n}}\right)^{k-1}\left|\bar{\Delta} T_{n}(s)\right|^{k}<\infty .
$$

In the special case, for $t_{n v}=p_{v} / P_{n},\left|T, p_{n}\right|_{k}$ summability is the same as $\left|\bar{N}, p_{n}\right|_{k}$ summability.

\section{The main result}

The object of this paper is to prove the following theorem.

THeOREM 2.1. Let $k \geq 1$. Let $\left(s_{n}\right)$ be a bounded sequence and suppose that $\left(\lambda_{n}\right)$ is a sequence such that

$$
\begin{gathered}
\sum_{n=0}^{m}\left(\frac{P_{n}}{p_{n}}\right)^{k-1}\left|\lambda_{n}\right|^{k}\left|t_{n n}\right|^{k}=O(1) \quad \text { as } m \longrightarrow \infty, \\
\sum_{n=0}^{m}\left|\Delta \lambda_{n}\right|=O(1) \text { as } m \longrightarrow \infty .
\end{gathered}
$$

If

$$
\begin{gathered}
\frac{1}{\left|t_{n n}\right|} \sum_{v=0}^{n-1}\left|\Delta_{v}\left(\hat{t}_{n v}\right)\right|=O(1) \quad \text { as } n \longrightarrow \infty \\
\sum_{n=v+1}^{m+1}\left(\frac{P_{n}}{p_{n}}\right)^{k-1}\left|\Delta_{v} \hat{t}_{n v}\right|\left|t_{n n}\right|^{k-1}=O\left(\left(\frac{P_{v}}{p_{v}}\right)^{k-1}\left|t_{v v}\right|^{k}\right) \quad \text { as } m \longrightarrow \infty,
\end{gathered}
$$




$$
\begin{gathered}
\frac{1}{\left|t_{n n}\right|} \sum_{v=0}^{n-1}\left|\Delta \lambda_{v}\right|\left|\hat{t}_{n, v+1}\right|=O(1) \quad \text { as } n \longrightarrow \infty, \\
\sum_{n=v+1}^{m+1}\left(\frac{P_{n}}{p_{n}}\right)^{k-1}\left|\hat{t}_{n, v+1}\right|\left|t_{n n}\right|^{k-1}=O(1) \quad \text { as } m \longrightarrow \infty,
\end{gathered}
$$

then the series $\sum a_{n} \lambda_{n}$ is summable $\left|T, p_{n}\right|_{k}$.

Proof. Let $\left(y_{n}\right)$ be the T-transform of the series $\sum a_{n} \lambda_{n}$. Then we have, by (1.6),

$$
Y_{n}=y_{n}-y_{n-1}=\sum_{v=0}^{n} \hat{t}_{n v} a_{v} \lambda_{v} .
$$

Since $\hat{t}_{n n}=t_{n n}$, by Abel's transformation, we get that

$$
\begin{aligned}
Y_{n} & =\sum_{v=0}^{n-1} \Delta_{v}\left(\hat{t}_{n v} \lambda_{v}\right) s_{v}+\hat{t}_{n n} \lambda_{n} s_{n} \\
& =\sum_{v=0}^{n-1} \Delta \lambda_{v} \hat{t}_{n, v+1} s_{v}+\sum_{v=0}^{n-1} \lambda_{v} \Delta_{v}\left(\hat{t}_{n v}\right) s_{v}+s_{n} t_{n n} \lambda_{n} \\
& =Y_{n}(1)+Y_{n}(2)+Y_{n}(3) .
\end{aligned}
$$

Using Minkowski's inequality, it is sufficient to show that

$$
\sum_{n=1}^{\infty}\left(\frac{P_{n}}{p_{n}}\right)^{k-1}\left|Y_{n}(r)\right|^{k}<\infty \quad \text { for } r=1,2,3 .
$$

Since $\left(s_{n}\right)$ is bounded, when $k>1$, applying Hölder's inequality with indices $k$ and $k^{\prime}$, where $1 / k+1 / k^{\prime}=1$, we have that

$$
\begin{aligned}
\sum_{n=1}^{m+1}\left(\frac{P_{n}}{p_{n}}\right)^{k-1}\left|Y_{n}(1)\right|^{k} \leq & \sum_{n=1}^{m+1}\left(\frac{P_{n}}{p_{n}}\right)^{k-1}\left\{\sum_{v=0}^{n-1}\left|\Delta \lambda_{v}\right|\left|\hat{t}_{n, v+1}\right|\left|s_{v}\right|\right\}^{k} \\
= & O(1) \sum_{n=1}^{m+1}\left(\frac{P_{n}}{p_{n}}\right)^{k-1} \sum_{v=0}^{n-1}\left|\Delta \lambda_{v}\right|\left|\hat{t}_{n, v+1}\right|\left|t_{n n}\right|^{k-1} \\
& \times\left\{\frac{1}{\left|t_{n n}\right|} \sum_{v=0}^{n-1}\left|\Delta \lambda_{v}\right|\left|\hat{t}_{n, v+1}\right|\right\}^{k-1} \\
= & O(1) \sum_{n=1}^{m+1}\left(\frac{P_{n}}{p_{n}}\right)^{k-1} \sum_{v=0}^{n-1}\left|\Delta \lambda_{v}\right|\left|\hat{t}_{n, v+1}\right|\left|t_{n n}\right|^{k-1} \\
= & O(1) \sum_{v=0}^{m}\left|\Delta \lambda_{v}\right| \sum_{n=v+1}^{m+1}\left(\frac{P_{n}}{p_{n}}\right)^{k-1}\left|\hat{t}_{n, v+1}\right|\left|t_{n n}\right|^{k-1} \\
= & O(1) \sum_{v=0}^{m}\left|\Delta \lambda_{v}\right|=O(1) \quad \text { as } m \longrightarrow \infty,
\end{aligned}
$$

by virtue of the hypothesis of Theorem 2.1. 
2520 On absolute matrix summability methods

Again using Hölder's inequality, we have

$$
\begin{aligned}
\sum_{n=1}^{m+1}\left(\frac{P_{n}}{p_{n}}\right)^{k-1}\left|Y_{n}(2)\right|^{k} & \leq \sum_{n=1}^{m+1}\left(\frac{P_{n}}{p_{n}}\right)^{k-1}\left\{\sum_{v=0}^{n-1}\left|\lambda_{v}\right|\left|\Delta_{v} \hat{t}_{n v}\right|\left|s_{v}\right|\right\}^{k} \\
= & O(1) \sum_{n=1}^{m+1}\left(\frac{P_{n}}{p_{n}}\right)^{k-1} \sum_{v=0}^{n-1}\left|\lambda_{v}\right|^{k}\left|\Delta_{v} \hat{t}_{n v}\right|\left|t_{n n}\right|^{k-1} \\
& \times\left\{\frac{1}{\left|t_{n n}\right|} \sum_{v=0}^{n-1}\left|\Delta_{v} \hat{t}_{n v}\right|\right\}^{k-1} \\
= & O(1) \sum_{n=1}^{m+1}\left(\frac{P_{n}}{p_{n}}\right)^{k-1} \sum_{v=0}^{n-1}\left|\lambda_{v}\right|^{k}\left|\Delta_{v} \hat{t}_{n v}\right|\left|t_{n n}\right|^{k-1} \\
= & O(1) \sum_{v=0}^{m}\left|\lambda_{v}\right|^{k} \sum_{n=v+1}^{m+1}\left(\frac{P_{n}}{p_{n}}\right)^{k-1}\left|\Delta_{v} \hat{t}_{n v}\right|\left|t_{n n}\right|^{k-1} \\
= & O(1) \sum_{v=0}^{m}\left(\frac{P_{v}}{p_{v}}\right)^{k-1}\left|\lambda_{v}\right|^{k}\left|t_{v v}\right|^{k}=O(1) \quad \text { as } m \longrightarrow \infty,
\end{aligned}
$$

by virtue of the hypothesis of Theorem 2.1.

Finally, we have that

$$
\sum_{n=1}^{m}\left(\frac{P_{n}}{p_{n}}\right)^{k-1}\left|Y_{n}(3)\right|^{k}=O(1) \sum_{n=1}^{m}\left(\frac{P_{n}}{p_{n}}\right)^{k-1}\left|t_{n n}\right|^{k}\left|\lambda_{n}\right|^{k}=O(1) \quad \text { as } m \longrightarrow \infty,
$$

by virtue of the hypothesis of Theorem 2.1.

Therefore, we get that

$$
\sum_{n=1}^{m}\left(\frac{P_{n}}{p_{n}}\right)^{k-1}\left|Y_{n}(r)\right|^{k}=O(1) \quad \text { as } m \longrightarrow \infty, \text { for } r=1,2,3 .
$$

This completes the proof of Theorem 2.1.

\section{An application}

Now we will prove the following corollary.

Corollary 3.1 (see [2]). Let $k \geq 1$. If the sequence $\left(s_{n}\right)$ is bounded and $\left(\lambda_{n}\right)$ is a sequence such that

$$
\begin{aligned}
\sum_{n=1}^{m} \frac{p_{n}}{P_{n}}\left|\lambda_{n}\right|^{k} & =O(1) \quad \text { as } m \longrightarrow \infty, \\
\sum_{n=1}^{m}\left|\Delta \lambda_{n}\right| & =O(1) \quad \text { as } m \longrightarrow \infty,
\end{aligned}
$$

then the series $\sum a_{n} \lambda_{n}$ is summable $\left|\bar{N}, p_{n}\right|_{k}$. 
Proof. In Theorem 2.1, let $t_{n v}=p_{v} / P_{n}$. Then to prove the corollary, it is sufficient to show that the conditions of Theorem 2.1 are satisfied.

If $t_{n n}=p_{n} / P_{n},(2.1)$ are automatically satisfied.

Since

$$
\begin{aligned}
\Delta_{v} \hat{t}_{n v} & =\hat{t}_{n v}-\hat{t}_{n, v+1} \\
& =\bar{t}_{n v}-\bar{t}_{n-1, v}-\bar{t}_{n, v+1}+\bar{t}_{n-1, v+1} \\
& =\sum_{i=v}^{n} t_{n i}-\sum_{i=v}^{n-1} t_{n-1, i}-\sum_{i=v+1}^{n} t_{n i}+\sum_{i=v+1}^{n-1} t_{n-1, i} \\
& =\frac{1}{P_{n}} \sum_{i=v}^{n} p_{i}-\frac{1}{P_{n-1}} \sum_{i=v}^{n-1} p_{i}-\frac{1}{P_{n}} \sum_{i=v+1}^{n} p_{i}+\frac{1}{P_{n-1}} \sum_{i=v+1}^{n-1} p_{i} \\
& =-\frac{p_{n} p_{v}}{P_{n} P_{n-1}},
\end{aligned}
$$

we get

$$
\frac{1}{\left|t_{n n}\right|} \sum_{v=0}^{n-1}\left|\Delta_{v} \hat{t}_{n v}\right|=\frac{P_{n}}{p_{n}} \sum_{v=0}^{n-1} \frac{p_{n} p_{v}}{P_{n} P_{n-1}}=O(1) \quad \text { as } n \longrightarrow \infty \text {. }
$$

Thus condition (2.2) is satisfied.

Using $\Delta_{v} \hat{t}_{n v}$ and $t_{n n}$,

$$
\begin{aligned}
\sum_{n=v+1}^{m+1}\left(\frac{P_{n}}{p_{n}}\right)^{k-1}\left|\Delta_{v} \hat{t}_{n v}\right|\left|t_{n n}\right|^{k-1} & =\sum_{n=v+1}^{m+1}\left(\frac{P_{n}}{p_{n}}\right)^{k-1} \frac{p_{n} p_{v}}{P_{n} P_{n-1}}\left(\frac{p_{n}}{P_{n}}\right)^{k-1} \\
& =p_{v} \sum_{n=v+1}^{m+1} \frac{p_{n}}{P_{n} P_{n-1}}=\frac{p_{v}}{P_{v}} \\
& =\left(\frac{P_{v}}{p_{v}}\right)^{k-1}\left|t_{v v}\right|^{k} \text { as } m \longrightarrow \infty,
\end{aligned}
$$

condition (2.3) is satisfied.

Since

$$
\begin{aligned}
\hat{t}_{n v} & =\bar{t}_{n v}-\bar{t}_{n-1, v}=\sum_{i=v}^{n} t_{n i}-\sum_{i=v}^{n-1} t_{n-1, i} \\
& =\frac{1}{P_{n}} \sum_{i=v}^{n} p_{i}-\frac{1}{P_{n-1}} \sum_{i=v}^{n-1} p_{i} \\
& =P_{v-1}\left(-\frac{1}{P_{n}}+\frac{1}{P_{n-1}}\right)=P_{v-1} \frac{p_{n}}{P_{n} P_{n-1}},
\end{aligned}
$$


2522 On absolute matrix summability methods

$$
\begin{aligned}
\frac{1}{\left|t_{n n}\right|} \sum_{v=0}^{n-1}\left|\Delta \lambda_{v}\right|\left|\hat{t}_{n, v+1}\right| & =\frac{P_{n}}{p_{n}} \sum_{v=0}^{n-1}\left|\Delta \lambda_{v}\right| P_{v} \frac{p_{n}}{P_{n} P_{n-1}} \\
& =\frac{1}{P_{n-1}} \sum_{v=0}^{n-1}\left|\Delta \lambda_{v}\right| P_{v}=O(1) \sum_{v=0}^{n-1}\left|\Delta \lambda_{v}\right|=O(1) \text { as } n \longrightarrow \infty,
\end{aligned}
$$

and condition (2.4) is satisfied.

Finally,

$$
\begin{aligned}
\sum_{n=v+1}^{m+1}\left(\frac{P_{n}}{p_{n}}\right)^{k-1}\left|\hat{t}_{n, v+1}\right|\left|t_{n n}\right|^{k-1} & =\sum_{n=v+1}^{m+1}\left(\frac{P_{n}}{p_{n}}\right)^{k-1} \frac{P_{v} p_{n}}{P_{n} P_{n-1}}\left(\frac{p_{n}}{P_{n}}\right)^{k-1} \\
& =P_{v} \sum_{n=v+1}^{m+1} \frac{p_{n}}{P_{n} P_{n-1}}=O(1) \quad \text { as } m \longrightarrow \infty
\end{aligned}
$$

so condition (2.5) is satisfied.

This completes the proof of the corollary.

\section{References}

[1] H. Bor, On two summability methods, Math. Proc. Cambridge Philos. Soc. 97 (1985), no. 1, $147-149$.

[2] _ A note on $\left|\bar{N}, p_{n}\right|_{k}$ summability factors, Rend. Mat. Appl. (7) 12 (1992), no. 4, 937-942 (1993).

[3] T. M. Flett, On an extension of absolute summability and some theorems of Littlewood and Paley, Proc. London Math. Soc. (3) 7 (1957), 113-141.

[4] G. H. Hardy, Divergent Series, Clarendon Press, Oxford University Press, New York, 1949.

[5] W. T. Sulaiman, Inclusion theorems for absolute matrix summability methods of an infinite series. IV, Indian J. Pure Appl. Math. 34 (2003), no. 11, 1547-1557.

H. S. Özarslan: Department of Mathematics, Faculty of Arts and Sciences, Erciyes University, 38039 Kayseri, Turkey

E-mail address: seyhan@erciyes.edu.tr

H. N. Öğdük: Department of Mathematics, Faculty of Arts and Sciences, Erciyes University, 38039 Kayseri, Turkey

E-mail address: nogduk@erciyes.edu.tr 


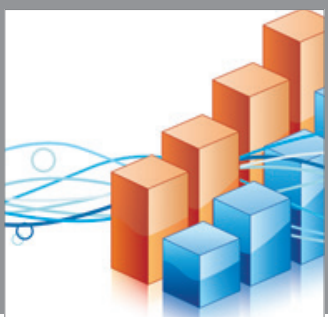

Advances in

Operations Research

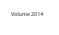

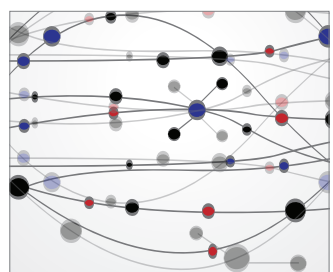

\section{The Scientific} World Journal
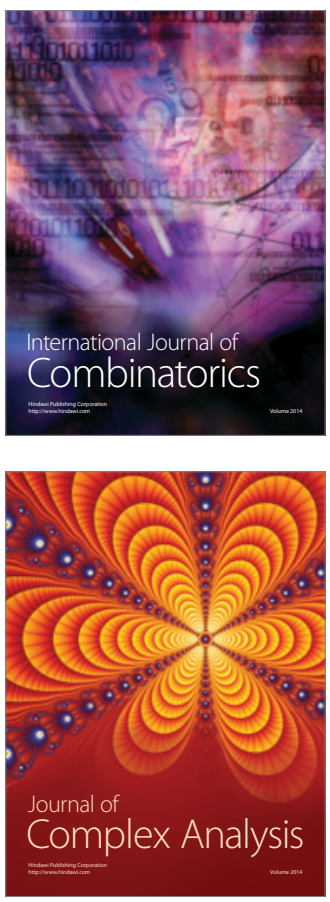

International Journal of

Mathematics and

Mathematical

Sciences
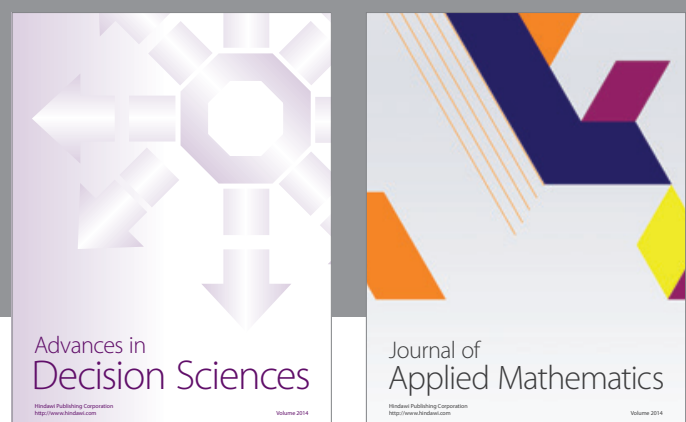

Journal of

Applied Mathematics
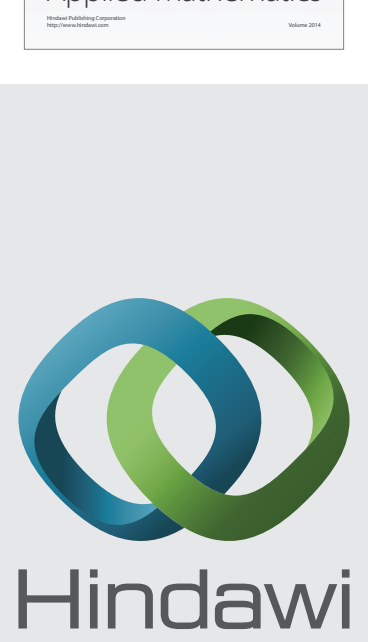

Submit your manuscripts at http://www.hindawi.com
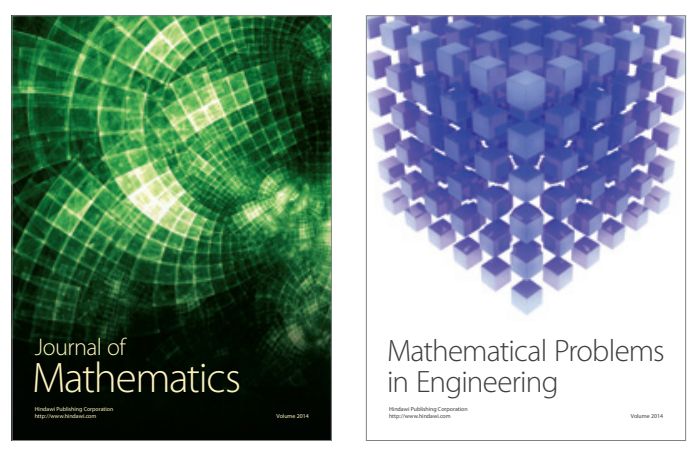

Mathematical Problems in Engineering
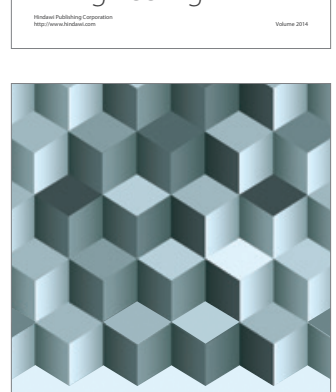

Journal of

Function Spaces
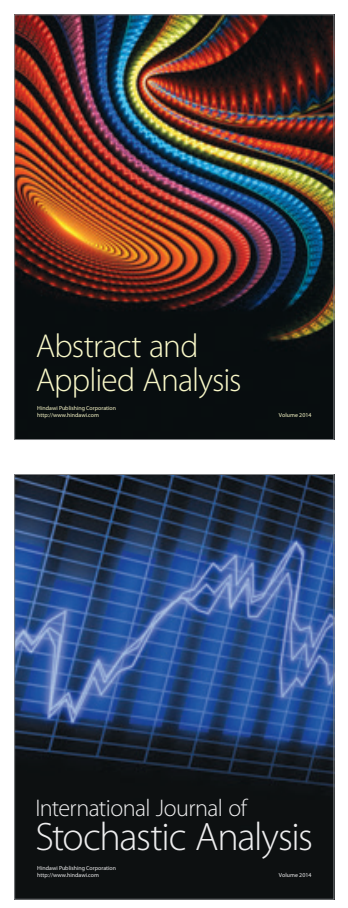

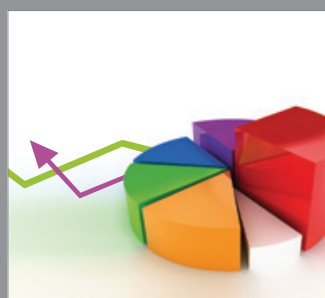

ournal of

Probability and Statistics

Promensencen
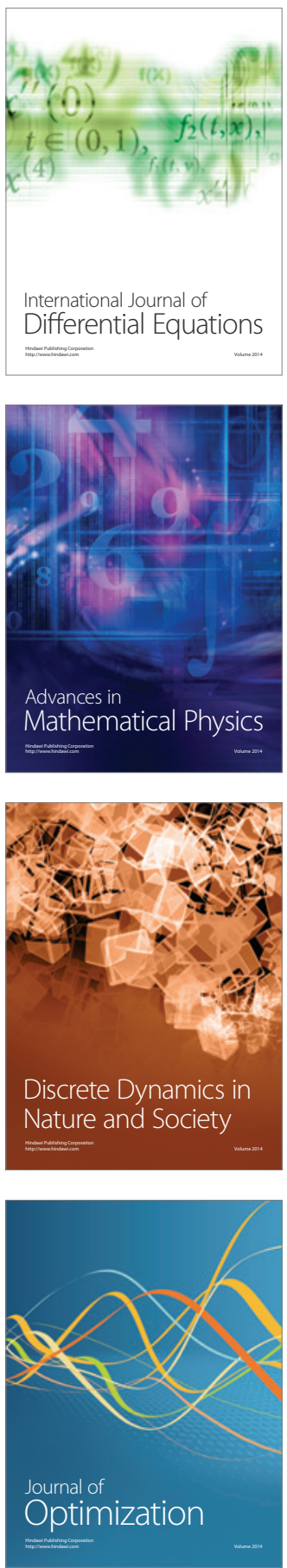